Trinity University

Digital Commons@ Trinity

Mathematics Faculty Research

Mathematics Department

$7-2010$

\title{
Population Models with Allee Effect: A New Model
}

Saber Elaydi

TrinityUniversity, selaydi@trinity.edu

Robert J. Sacker

Follow this and additional works at: https://digitalcommons.trinity.edu/math_faculty

Part of the Mathematics Commons

\section{Repository Citation}

Elaydi, S.N., \& Sacker, R.J. (2010). Population models with Allee effect: A new model. Journal of Biological Dynamics, 4(4), 397-408. doi: $10.1080 / 17513750903377434$

This Post-Print is brought to you for free and open access by the Mathematics Department at Digital Commons @ Trinity. It has been accepted for inclusion in Mathematics Faculty Research by an authorized administrator of Digital Commons @ Trinity. For more information, please contact jcostanz@trinity.edu. 


\title{
Population Models with Allee Effect: A New Model
}

\author{
Saber N. Elaydi ${ }^{\S}$ and Robert J. Sacker ${ }^{* \dagger}$ \\ $\nmid$ †epartment of Mathematics, University of Southern California, Los Angeles, CA 90089-2532 USA \\ $\ddagger$ Department of Mathematics, Trinity University, San Antonio, TX 78212 USA
}

(00 December 2008)

\begin{abstract}
In this paper we develop several mathematical models of Allee effects. We start by defining the Allee effect as a phenomenon in which individual fitness increases with increasing density. Based on this biological assumption, we develop several fitness functions that produce corresponding models with Allee effects. In particular, a rational fitness function yields a new mathematical model that is our focus of study. Then we study the dynamics of 2-periodic systems with Allee effects and show the existence of an asymptotically stable 2-periodic carrying capacity.
\end{abstract}

Keywords: Allee effect, fitness function, carrying capacity, threshold point, stability.

AMS Subject Classification: 39A11, 92D25

\section{Introduction}

In the last decade, there has been a renewed interest in a biological phenomenon called the Allee effect. A biology book [4] just appeared that is solely dedicated to study this phenomenon and hundreds of papers dealing with the Allee effect were cited in this book. Surprisingly, the literature on mathematical modeling of the Allee effect is lagging behind. For the convenience of the reader we cite some of the recent mathematical papers on modeling the Allee effect: [5], [15], [18], [21], [24], [25].

But what is the Allee effect? The Allee effect is a phenomenon in population dynamics attributed to the biologist Warder Clyde Allee [1,2]. Allee proposed that the per capita birth rate declines at low population densities. Under such scenario, a population at low densities may slide into extinction. Allee found that the highest per capita growth rates of the population of the flour beetles, Tribolium cofusum, were at intermediate densities. Moreover, when fewer mates were available, the females produced fewer eggs, a rather unexpected outcome. Allee did not provide a definite and precise definition of this new notion. Stephens, Sutherland, and Freckleton [22] defined the Allee effect as "a positive relationship between any component of individual fitness and either numbers or density of conspecifics." In classical dynamics, we have a negative density dependence, that is, fitness decreases with increasing density. The Allee effect, however, produces a positive density dependence, that is, fitness increases with increasing density.

The instability of the lower equilibrium (Allee threshold) [4] means that natural populations subject to a demographic Allee effect are unlikely to persist in the range of population sizes where the effect is manifest. Another issue that may cause some confusion in the use of the term Allee effect whether the phenomenon is caused by low population sizes or by low population densities. Though for field ecologists, a drop in number will be inseparable from a corresponding reduction in density. Allee himself considered both types of Allee effect and observed the Allee effect caused by reduction in the number of mice, and the Allee effect caused by the reduction in density of flour beetles, Tribolium confusum [1]. In [23], Stephens and Sutherland described several scenarios that cause the Allee effect in both animals and plants. For

\footnotetext{
$\S$ Supported by Trinity University Faculty Development Grant.

*Corresponding author. Email: rsacker@usc.edu, http://rcf.usc.edu/ rsacker

$\dagger$ Supported by University of Southern California, Letters Arts and Sciences Faculty Development Grant.
} 
example, cod and many freshwater fish species have high juvenile mortality when there are fewer adults. While fewer red sea urchin give rise to worsening feeding conditions of their young and less protection from predation. In some mast flowering trees, such as Spartina alterniflora, with a low density have a lower probability of pollen grain finding stigma in wind-pollinated plants In [23].

The main aim of this paper is to derive various single-species models with Allee effects based on sound biological assumptions. Starting with the assumption that individual fitness increases for small population densities, we develop a variety of fitness functions which produce corresponding models with Allee effects.

In the sequel, we will consider the difference equation

$$
x_{t+1}=f\left(x_{t}\right) \quad f: \mathbb{R}^{+} \rightarrow \mathbb{R}^{+}=[0, \infty), \quad t=0,1.2, \ldots
$$

whose dynamics exhibit the Allee effect and in certain cases obtain some new results. Some of the models are modifications of old, well studied models while others are new models introduced to give the modeler the ability to take into account effects not available from older models. We must leave it to others to glean whatever benefit can be had from looking at these new models. Any function $f$ whose graph passes through the origin and remains below the diagonal near zero and later crosses the diagonal twice will give rise to the Alee effect. For simplicity in discussion we will refer to the first crossing as the Allee threshold, $A$ and the later crossing as the carrying capacity, $K$.

In investigating certain phenomena such as resonance or attenuation it is advantageous to have the carrying capacity $K$ as a parameter in the model. For example the Cushing-Henson conjecture [6,7] for the Beverton-Holt equation

$$
x_{t+1}=\frac{\mu K x_{t}}{K+(\mu-1) x_{t}}
$$

stated that a periodically varying environment $\left\{K_{0}, K_{1}, \ldots, K_{p-1}, K_{p}=K_{0}\right\},(1)$ gives rise to a periodic state $\left\{\hat{x}_{0}, \hat{x}_{1}, \ldots, \hat{x}_{p-1}, \hat{x}_{p}=\hat{x}_{0}\right\}$ that globally attracts all positive solutions and $(2)$, the average

$$
A v\left(\bar{x}_{t}\right) \doteq \frac{1}{p} \sum_{i=0}^{p-1} \bar{x}_{i}
$$

satisfies $\operatorname{Av}\left(\bar{x}_{t}\right)<\operatorname{Av}\left(K_{t}\right)$, i.e. there is attenuation. Cushing and Henson established these fact for $p=2$ and later, $[9,12,17]$ solved the general case by a variety of different methods. In [13] it was established in the case of randomly varying $K$. In [11] it was also shown by the authors (for $p=2$ ) that if the growth parameter $\mu$ is allowed to vary as well then the opposite inequality in the averages can occur, namely resonance, if the parameters are sufficiently in phase.

The existence of the periodic solution follows directly from the concavity of the functions

$$
x_{t+1}=\frac{\mu K_{t} x_{t}}{K_{t}+(\mu-1) x_{t}}
$$

and the fact their graphs cross the diagonal [9] since the class of such functions form a semigroup under composition. Further, if the fixed points $x_{f}$ and $x_{g}$ of $f$ and $g$ satisfy $x_{f}<x_{g}$ then the fixed points of the compositions, $x_{f \circ g}$ and $x_{g \circ f}$ lie strictly between

$$
x_{f}<x_{f \circ g}<x_{g}, \quad x_{f}<x_{g \circ f}<x_{g},
$$

the containment property. From this it follows that the periodic state lies inside the envelope of the fixed points of the component maps.

What is interesting is that the same containment property holds if the functions are increasing and convex and can thus be applied to most of the models displaying the Allee effect since their functions are 


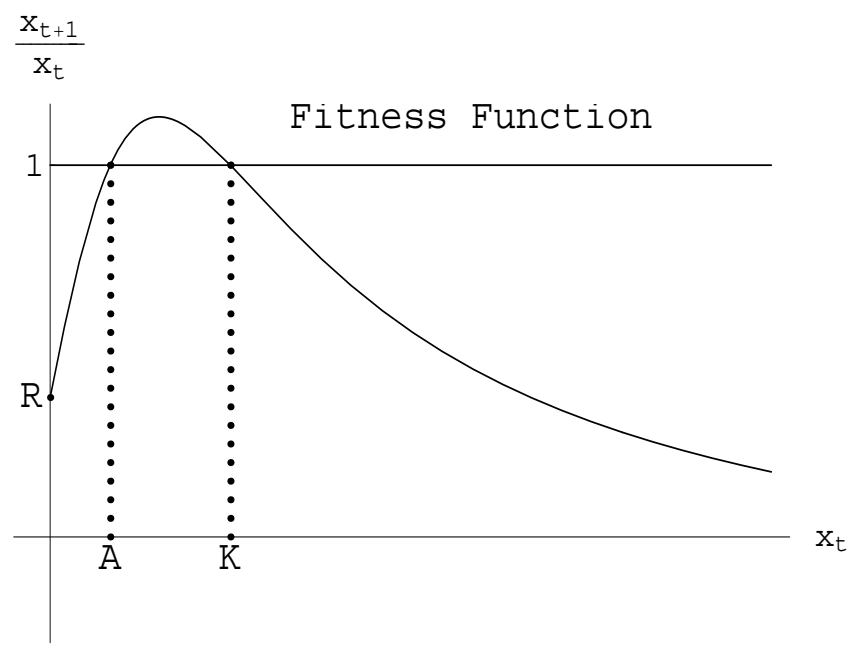

Figure 1. Fitness Function

convex in some neighborhood of the origin. Thus let us define

$$
\begin{aligned}
\mathcal{F}_{0} \doteq & \left\{f: \mathbb{R}^{+} \rightarrow \mathbb{R}^{+} \mid f(0)=0, f \uparrow\right. \text { and } \\
& \left.f \text { convex on some interval }\left[0, b_{f}\right]\right\}, \\
\mathcal{F}_{A} \doteq & \left\{f \in \mathcal{F}_{0} \mid \exists x_{1}, x_{2} \in\left[0, b_{f}\right] \ni\right. \\
& \left.f\left(x_{1}\right)<x_{1}, f\left(x_{2}\right)>x_{2}\right\}, \\
\mathcal{F}_{\mathcal{K}} \doteq & \left\{f \in \mathcal{F}_{A} \mid \exists \text { unique } K_{f}>b_{f} \ni f\left(K_{f}\right)=K_{f}\right\} .
\end{aligned}
$$

Then $f \in \mathcal{F}_{A}$ implies that $f$ has a unique Allee fixed point $A_{f}$ in $\left[0, b_{f}\right]$. The following theorem can then be proved

THEOREM 1.1 Let $\mathcal{U} \subset \mathcal{F}_{\mathcal{K}}$ be a subset such that

$$
\begin{gathered}
\sup _{f \in \mathcal{U}} A_{f}<\inf _{f \in \mathcal{U}} K_{f}, \quad \text { then } f, g \in \mathcal{U} \Longrightarrow \\
A_{f}<A_{f \circ g}<A_{g} \quad \text { and } \quad A_{f}<A_{g \circ f}<A_{g} .
\end{gathered}
$$

and $\mathcal{U}$ is a semigroup under composition.

Proof Since $f$ and $g$ are convex, it follows that $g \circ f$ is also convex. Hence $A_{f}<A_{g} \Longrightarrow g\left(A_{f}\right)<A_{f}$ and $A_{g}<f\left(A_{g}\right)$. Therefore, $g \circ f\left(A_{f}\right)=g\left(A_{f}\right)<A_{f}$. Choose $z$ such that $f(z)=A_{g}$ and $A_{f}<z<A_{g}$. Then $g(f(z))=A_{g}>z$. By the intermediate value theorem, there exists a fixed point $A_{g \circ f}$ between $A_{f}$ and $A_{g}$. Similarly, $f \circ g\left(A_{f}\right)<f\left(A_{f}\right)=A_{f}$, and $f \circ g\left(A_{g}\right)=f\left(A_{g}\right)>A_{g}$.

From the above we see that it is important that the carrying capacity, growth rate, or threshold point appear in the model as parameters if we want to consider their respective variations with time (periodic, stochastic, almost periodic, etc.).

As an aid in the construction of models we define the fitness function

$$
u(x)=\frac{x_{t+1}}{x_{t}}
$$

as a function that passes through the points $(0, R),(A, 1),(K, 1)$, where $R$ is the repulsion $(R>1)$ or attraction $(R<1)$ rate at 0 . 
When the population is sufficiently small (close to zero), the competition is negligible and the net reproductive rate can be approximated by $R$. Thus $\frac{x_{t+1}}{x_{t}}=R$ when $x_{t}$ is close to zero. This gives us the point $(0, R)$ in the graph $\frac{x_{t+1}}{x_{t}}$ versus $x_{t}$. When the population grows larger, intra-specific competition becomes significant and at some population sizes $x_{t}=A$, and $x_{t}=K$, the birth and death rates are equal and thus $\frac{x_{t+1}}{x_{t}}=1$. This gives us the points $(A, 1)$ and the point $(K, 1)$. Then we join these three points by a suitable curve (Figure 1). The fixed point $A$ will be called the threshold point and the fixed point $K$ will be called the carrying capacity.

\section{The Exponential Model}

By letting

$$
u(x)=e^{-r\left(1-\frac{x}{A}\right)\left(1-\frac{x}{K}\right)}, \quad r>0
$$

we obtain $x_{t+1}=f\left(x_{t} ; r\right)$ with

$$
f(x ; r)=x e^{-r\left(1-\frac{x}{A}\right)\left(1-\frac{x}{K}\right)} .
$$

Besides $A$ and $K$ the additional parameter is $r$ and $e^{-r}$ is the rate of attraction at 0 . Introducing the repulsion rate $\mu \doteq f^{\prime}(A ; r)$ at the threshold point $\mathrm{A}$, one obtains, one obtains the difference equation $x_{t+1}=G\left(x_{t} ; \mu\right)$ where

$$
G(x ; \mu)=x e^{(1-\mu)(A-x)(K-x) / A(K-A)}
$$

or introducing the attraction rate $\lambda \doteq F^{\prime}(K ; r)$ at the carrying capacity $K$, one obtains the difference equation $x_{t+1}=H\left(x_{t} ; \lambda\right)$ where

$$
H(x ; \lambda)=x e^{(\lambda-1)(A-x)(K-x) / K(K-A)} .
$$

\section{The $\lambda$-Ricker Map}

In this section we obtain some properties of a modification of the Ricker map that possesses the Allee effect. The development here follows closely the argument given in [19]. In [19] the case $\lambda=1$ (the classic Ricker equation) and $p_{j} \in(0,2), j=0,1, \ldots, k-1$ was carried out and it was shown that (3.2) always has a periodic solution $\left\{\hat{x}_{0}, \hat{x}_{1}, \ldots, \hat{x}_{k-1}, \hat{x}_{k}=\hat{x}_{0}\right\}$ that globally attracts all positive solutions and there is neither attenuation nor resonance, i.e. one has $\operatorname{Av}\left(\bar{x}_{j}\right)=\operatorname{Av}\left(p_{j}\right)$.

Consider the $\lambda$-Ricker map and its derivative

$$
\begin{aligned}
& R_{p}(x)=x^{\lambda} e^{p-x}, \quad x, p \in \mathbb{R}^{+}, \quad \lambda>1 \\
& R_{p}^{\prime}(x)=x^{\lambda-1}(\lambda-x) e^{p-x}
\end{aligned}
$$

(i) Notice that the fixed point 0 is always asymptotically stable since $R_{p}^{\prime}(0)=0$,

(ii) The threshold point $A$ is always unstable if it exists $(p>1)$. This may be proved by a simple argument [8].

(iii) The carrying capacity $K$ is asymptotically stable for $1<p<3-\ln 3$ If $p=3-\ln 3$, the carrying capacity $K=3$ is still asymptotically stable since the Schwarzian derivative $S R_{p}<0$, [8]. As $p$ passes the value $3-\ln 3$, a period doubling bifurcation begins as $R_{p}^{\prime}(3)=-1$. Numerical computation shows that the new born 2 -periodic orbit is asymptotically stable for $3-\ln 3<p<\approx 2.35$. The perioddoubling scenario continues until $p \approx 2.235$ after which we enter the chaotic region. Now for $p \geq 3$ the chaotic region disappears and the fixed point 0 becomes globally asymptotically stable. For $p=3$, 
the threshold point is $A \approx 0.0524$. Note that for the critical point $c=2, R_{p}^{2}(2)<0.524$ and hence it converges to 0 .

Now consider the $k$-periodic sequence $\left\{p_{0}, p_{1}, \ldots, p_{k-1}\right\}$ with $p_{i+k}=p_{i}$, and the $k$-periodic difference equation

$$
x_{n+1}=R_{p_{n}}\left(x_{n}\right)=x_{n}^{\lambda} e^{p_{n}-x_{n}}, \quad n=0,1, \ldots
$$

Now consider the composition

$$
\Phi_{m}=R_{p_{m-1}} \circ R_{p_{m-2}} \circ \cdots \circ R_{p_{1}} \circ R_{p_{0}}
$$

For any $m$-periodic orbit

$$
\left\{x_{0}, x_{1}, \ldots, x_{m}\right\} \subset \mathcal{I}, \quad x_{m}=x_{0}
$$

we have from (3.1),

$$
R_{p_{n}}^{\prime}\left(x_{n}\right)=x_{n}^{\lambda-1}\left(\lambda-x_{n}\right) e^{p_{n}-x_{n}}=\frac{\lambda-x_{n}}{x_{n}} x_{n}^{\lambda} e^{p_{n}-x_{n}}=\left(\lambda-x_{n}\right) \frac{x_{n+1}}{x_{n}}
$$

Taking the derivative of $(3.3)$,

$$
\begin{aligned}
\left|\Phi_{m}^{\prime}\left(x_{0}\right)\right| & =\left|R_{p_{k-1}}^{\prime}\left(x_{k-1}\right) R_{p_{k-2}}^{\prime}\left(x_{k-2}\right) \times \cdots \times R_{p_{0}}^{\prime}\left(x_{0}\right)\right| \\
& =\left|\frac{x_{m}}{x_{0}}\right| \prod_{i=0}^{m-1}\left|\lambda-x_{i}\right|=\prod_{i=0}^{m-1}\left|\lambda-x_{i}\right| .
\end{aligned}
$$

The next step is to find an invariant interval $\mathcal{I}$ on the $x$-axis on which $\left|\Phi_{k}^{\prime}(x)\right|<1$. Consider only the case $\lambda=2$ and define $\bar{p}=3-\ln 3$. Then the right endpoint of $\mathcal{I}$ can be defined as $R_{\bar{p}}(2)=4 e^{1-\ln 3}=\frac{4}{3} e \approx 3.6244$ since each function $R_{p}$ attains its maximum value at $x=2$.

In defining the left endpoint, there is a subtle distinction between the period $k>2$ and $k=2$ and in order to appreciate this let us recall that for $k=1$, as $p$ increases through 1 the fixed points constituting the Allee threshold $A$ and the carrying capacity $K$ are created through a saddle-node bifurcation so that at $p=1, A=1=K$, for $p>1$ we have $A<1<K$ and as $p$ increases further, $A$ and $K$ move monotonically away from 1 .

\subsection{Invariant Interval $(\lambda=2$, Period $k>2$ case)}

What we will show in that for $p \in(1,3-\ln 3)$ the invariant interval $\mathcal{I}=\left[1, R_{\bar{p}}(2)\right]$ but only after making one further restriction on the variation of the adjacent $p$ values, i.e. the $p$ values of adjacent maps $R_{j} \circ R_{j-1}$ in (3.3). To see why this is needed we consider points very close to the endpoints of the $p$-interval, $p_{0}=\bar{p}-0.001$ and $p_{1}=1.001$ in (3.3). A calculation shows

$$
x_{2}=R_{p_{1}} \circ R_{p_{0}}(2) \approx 0.95467
$$

Now let $A_{p}$ denote the Allee threshold of $R_{p}$ and choose $p_{2}, p_{3}, \ldots, p_{k-1}$ so close to 1 that $x_{2} \in\left(0, A_{p}\right)$ for all $p \in\left\{p_{2}, p_{3}, \ldots, p_{k-1}\right\}$. Then let $k$ be so large that $x_{k}=R_{p_{k-1}}\left(x_{k-1}\right) \in\left(0, A_{p_{0}}\right)$, the smallest of the "Allee intervals". Then the orbit $\left\{x_{n}\right\}$ starting at $x_{0}=2$ will go extinct, $\lim _{n \rightarrow \infty} x_{n}=0$. 


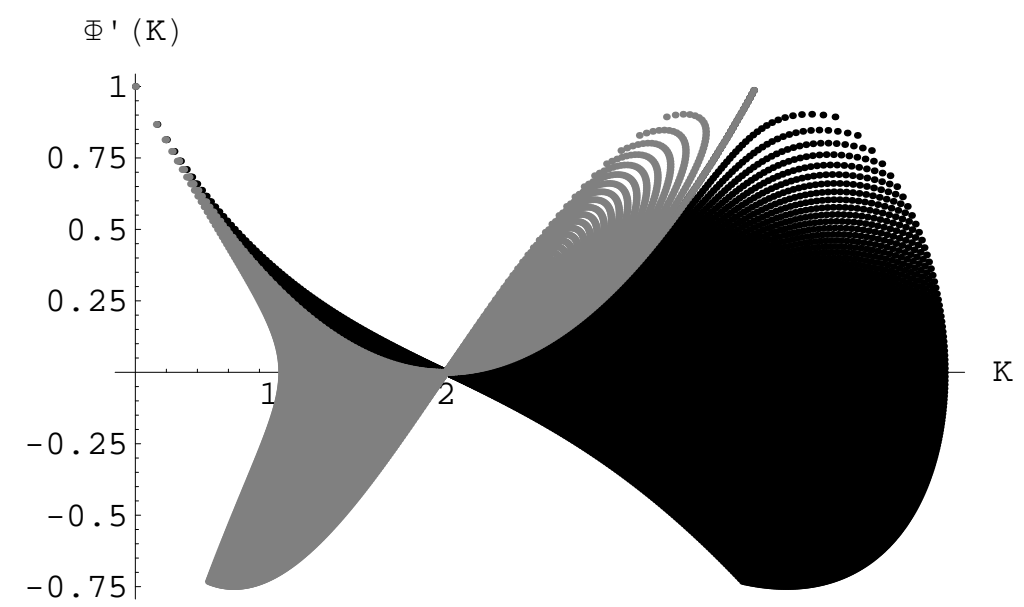

Figure 2. The black region shows the values of the derivatives of $\Phi$ at the carrying capacity of $f \circ g$, while the grey region depicts the values of the derivatives of $\widetilde{\Phi}$ at the carrying capacity of $g \circ f$

However, if we restrict the variation

$$
\max \left|p_{j}-p_{j-1}\right|<0.8523
$$

it can be verified numerically that in $(3.5), x_{2}>1>A_{p}$ for all $p \in[1,3-\log 3]$. Note: The length of the allowable $p$ interval is $\approx .90138$ so the above restriction on the allowable variation between the $p$ values of successive maps doesn't seem too restrictive.

To see that $\left|\Phi_{m}^{\prime}(x)\right|<1$ for $x \in \mathcal{I}$ we argue as in [19] and assume one of the factors $\left|2-x_{j}\right| \geq 1$ in (3.4). Now consider the product

$$
\left|\left(2-x_{j-1}\right)\left(2-x_{j}\right)\right|=\left|(2-x)\left(2-x^{2} e^{p-x}\right)\right|, \quad x=x_{j-1},
$$

and define

$$
g(x)=(2-x)\left(2-x^{2} e^{p-x}\right)
$$

or $1 \leq p \leq 3-\ln 3$ and $x \in \mathcal{I}$, g attains its maximum value 1 at $x=3$ and $p=3-\ln 3$. Further, the maximum value is $<1$ whenever $p<3-\ln 3$ and $x \in \mathcal{I}$. Another solution of $g^{\prime}(x)=0$ is computed numerically and is approximated by $u=1.315166$. And for $p=3-\ln 3, g(u)=-0.759154$. This is demonstrated clearly in figures 2 and 3.

Thus, we have shown that the interval $\mathcal{I}$ is invariant under the mapping $\Phi_{k}$ for $1<p<3-\ln 3$ and therefore there is at least one fixed point.

\subsection{Invariant Interval $(\lambda=2$, Period $k=2$ case $)$}

Here the restriction on the $p$-variation is not needed since although $x_{2}<1$, the next function to act is $R_{p_{0}}$ and $R_{p_{0}}\left(x_{2}\right) \approx 2.3465$. Thus, using the endpoints of the $p$-interval we define the invariant interval

$$
\mathcal{I}=[a, b], \quad \text { where } \quad b=R_{\bar{p}}(2) \approx 3.6244, \quad \text { and } \quad a=R_{1}(b) \approx 0.9522 .
$$

Again, the argument given above for the function $g$ defined in $(3.6)$, gives $\left|\left(2-x_{j-1}\right)\left(2-x_{j}\right)\right|<1$. 


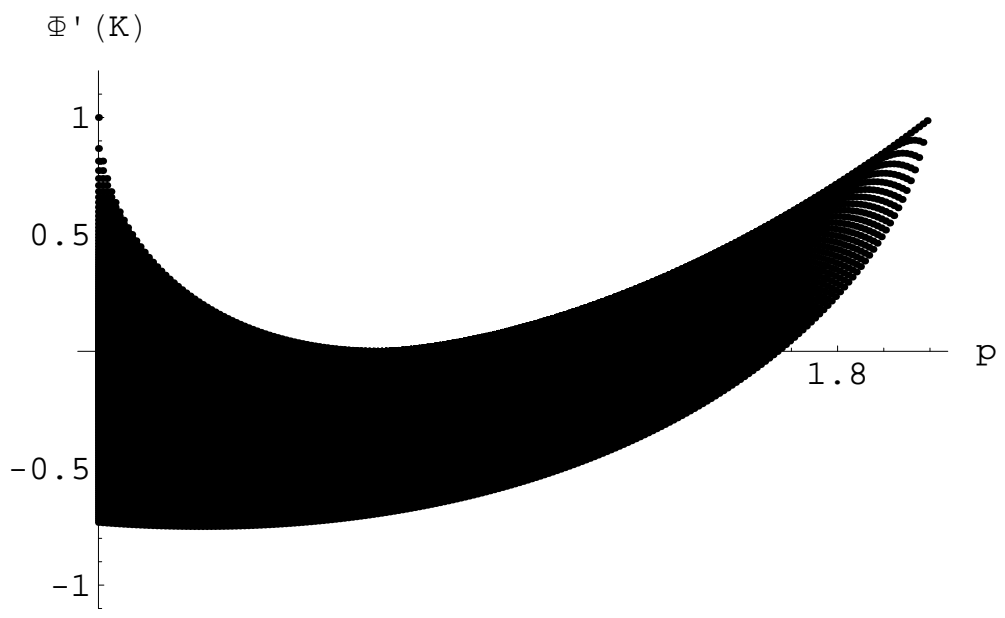

Figure 3. The black region represents the values of the derivatives of $\Phi$ and $\widetilde{\Phi}$ at the carrying capacity of $f \circ g$ and $g \circ f$ when the parameter $p_{0}$ and $p_{1}$ vary between 1 and $3-\ln 3$

\section{3. $\quad$ Asymptotic Stability of Fixed Point}

From (3.4) it follows that any fixed point in the invariant interval $\mathcal{I}$ is asymptotically stable. From this it follows that the fixed point is unique and attracts all orbits in $\mathcal{I}$, see [8, p.52, Th. 1.30]. This, in turn, implies that $m=k$, i.e. the periodic orbit has the same period as the mapping system.

\section{The Exponential Allee Model}

By defining the fitness function to be

$$
u(x)=e^{-r\left(1-\frac{x}{A}\right)\left(1-\frac{x}{K}\right)}, \quad r>0
$$

we obtain the difference equation $x_{t+1}=f\left(x_{t} ; r\right)$ with

$$
f(x ; r)=x e^{-r\left(1-\frac{x}{A}\right)\left(1-\frac{x}{K}\right)} .
$$

Besides $A$ and $K$, the additional parameter is $r$ and $e^{-r}$ is the rate of attraction at 0 .

Introducing the repulsion rate at the threshold point $A, \mu \doteq f^{\prime}(A ; r)$, one obtains the difference equation $x_{t+1}=G\left(x_{t} ; \mu\right)$ where

$$
G(x ; \mu)=x e^{(1-\mu)(A-x)(K-x) / A(K-A)}
$$

or introducing the attraction rate at the Carrying capacity $K, \lambda \doteq F^{\prime}(K ; r)$, one obtains the difference equation $x_{t+1}=H\left(x_{t} ; \lambda\right)$ where

$$
H(x ; \lambda)=x e^{(\lambda-1)(A-x)(K-x) / K(K-A)} .
$$

\section{A Canonical Form}

In this section we study the following fractional quadratic map

$$
f(x)=\frac{x}{\alpha+x} \frac{x}{p+q x} .
$$


By proper choice of $p$ and $q$ one obtains

$$
F(x)=\frac{x}{\alpha+x} \frac{(\alpha+A)(\alpha+K) x}{A K+\alpha x}
$$

In this form it follows that

$$
f(0)=0, \quad f(A)=A \quad \text { and } \quad f(K)=K .
$$

Also,

$$
f^{\prime}(x)=\frac{(\alpha+A)(\alpha+K)\left(2 \alpha A K+\alpha^{2} x+A K x\right) x}{(\alpha+x)^{2}(A K+\alpha x)^{2}}
$$

and since $A$ and $K$ occur symmetrically in (5.1) we assume without loss of generality that $A<K$. Direct computation shows that $f^{\prime}(0)=0$ and

$$
f^{\prime}(A)=\frac{2 \alpha K+\alpha^{2}+A K}{(\alpha+A)(\alpha+K)}>1>f^{\prime}(K)=\frac{2 \alpha A+\alpha^{2}+A K}{(\alpha+A)(\alpha+K)} .
$$

Thus for the difference equation

$$
x_{n+1}=f\left(x_{n}\right),
$$

the origin is the attracting extinction state, $A$ is the repelling, or Allee threshold and $K$ is the attracting fixed point or carrying capacity.

Note also that the mean

$$
\frac{f^{\prime}(A)+f^{\prime}(K)}{2}=1
$$

\section{A New Model}

In this section we develop a general model of populations with the Allee effects. By a suitable choice of the parameters, one may be able to generate maps with the same qualitative dynamics as the previously discussed maps. Moreover, this new model may produce a Beverton-Holt type map with an Allee effect. The authors believe this new model is the right model for populations that are under the influence of the Allee effects. The model also allows one to study the dynamics of the map after a large surge (overstocking) in the population. For maps of the Ricker type, if the surge is large enough the population will always go extinct, whereas for maps of the Beverton-Holt type (concave) the population always returns toward the carrying capacity. In the following map there is an additional parameter " $b$ " which we call the shock recovery parameter which controls this effect.

Consider the fitness function of the form

$$
u(x)=\frac{d x+e}{x^{2}+b x+c} .
$$

Forcing the graph of $u$ to pass through the three points in Figure 1 we obtain

$$
u(x)=\frac{(A+K+b) x+R \frac{A K}{1-R}}{x^{2}+b x+\frac{A K}{1-R}}
$$


and the corresponding difference equation $x_{t+1}=f\left(x_{t} ; R\right)=x_{t} u\left(x_{t}\right)$, or

$$
f\left(x_{t} ; R\right)=x_{t} \frac{(A+K+b) x_{t}+R \frac{A K}{1-R}}{x_{t}^{2}+b x_{t}+\frac{A K}{1-R}}
$$

where $b$ is still a free parameter. The parameter $R$ is the attraction rate at the extinction point 0 . Introducing the growth rate $\mu$ at the Allee threshold, $\mu=f^{\prime}(A ; R)$ and using this to eliminate $R$ one obtains the difference equation $x_{t+1}=G\left(x_{t} ; \mu\right)$ where

$$
G(x ; \mu) \doteq x \frac{(A+K+b) x+\frac{A}{(\mu-1)}[2 K+b-\mu(A+K+b)]}{x^{2}+b x+\frac{A}{(\mu-1)}[K-\mu A-(\mu-1) b]} .
$$

Then we have

$$
\begin{gathered}
f(0 ; R)=G(0 ; \mu)=0, \quad f(A, R)=G(A, \mu)=A, \\
f(K ; R)=G(K, \mu)=K, \quad f^{\prime}(0 ; R)=R, \quad G^{\prime}(A, \mu)=\mu \\
\lim _{x \rightarrow \infty} f(x)=A+K+b .
\end{gathered}
$$

Parameter $b$ regulates the asymptotic value at $\infty$.

For $b=0$

$$
G(x ; \mu)=\frac{(A+K) x^{2}+\frac{A}{(\mu-1)}[2 K-\mu(A+K)] x}{x^{2}+\frac{A}{(\mu-1)}[K-\mu A]} .
$$

Since

$$
f\left(x_{t}, R\right)=f(x)=x u(x)
$$

it follows that

$$
f^{\prime}(x)=u(x)+x u^{\prime}(x)
$$

Moreover,

$$
u^{\prime}(x)=(R-1) \frac{(R-1)(A+K+b) x^{2}+2 R A K x+(b R-A-K-b)}{\left[(1-R) x^{2}+b(1-R) x+A K\right]^{2}} .
$$

Since $A+K+b \geq 0, u^{\prime}(x)=0$ has at most one positive real root $C_{u}$ as shown in figure 1 .

Notice that $u(x)>0$. By a simple computation one concludes that $u^{\prime \prime}(x)<0$ on $(0, \infty)$. Thus $u(x)$ is concave on $(0, \infty)$. Moreover, $f^{\prime}(x)=u(x)+x u^{\prime}(x) \geq 0$ on $\left(0, C_{u}\right)$. Notice also that $f(x)$ is decreasing on $\left(C_{u}, \infty\right)$.

To insure that $f^{\prime}(K) \geq 0$ on $[0, K]$, we require that $f^{\prime}(K)=u(K)+K u^{\prime}(K) \geq 0$. This implies that

$$
b \geq-A\left(\frac{2-R}{1-R}\right)
$$

Standing assumption: In the sequel, (6.6) is always assumed.

Under this assumption $f(x)$ is increasing on $[0, K]$. In this case, it is easy to prove the following result 
THEOREM 6.1 Under assumption (6.6) we have the following

(i) $x^{*}=0$ is locally asymptotically stable,

(ii) $x^{*}=A$ is unstable,

(iii) $x^{*}=K$ is locally asymptotically stable.

Proof See [8].

If in addition, $A+K+b \geq A$ or $b \geq-K$, then $\mathcal{B}(0)=[0, A)$ and $\mathcal{B}(K)=[A, \infty)$, where $\mathcal{B}$ denotes the basin of attraction.

\section{New class of maps}

Let us define a subset of $\mathcal{F}_{K}$,

$$
\mathcal{F}=\left\{h \in \mathcal{F}_{K} \mid h \uparrow \text { and concave on }\left[b_{h}, K_{h}\right] \text { and h has at most one critical point } \operatorname{in}\left[K_{h}, \infty\right)\right\}
$$

Lemma 7.1 Let $\mathcal{W}$ be a subset of $\mathcal{F}$ such that

$$
\sup _{h \in \mathcal{W}} A_{h}<\inf _{h \in \mathcal{W}} K_{h}
$$

Let $f, g \in \mathcal{W}$, with $K_{f}<K_{g} \Rightarrow$. Then

(i) $K_{f}<K_{f \circ g}<K_{g}$

(ii) $K_{f}<K_{g \circ f}<K_{g}$.

Proof $g\left(f\left(K_{f}\right)\right)=g\left(K_{f}\right)>K_{f}$ and $g\left(f\left(K_{g}\right)\right)<g\left(K_{g}\right)=K_{g}$. Hence there exists a fixed point $K_{g \circ f}$ of $g \circ f$ with $K_{f}<K_{g \circ f}<K g$. Now $f\left(g\left(K_{g}\right)\right)=f\left(K_{g}\right)<K_{g}$. Moreover, since $g\left(K_{f}\right)>K_{f}$, it follows that $f\left(g\left(K_{f}\right)\right)>K_{f}$. Hence there exists a fixed point $K_{f \circ g}$ of $f \circ g$ with $K_{f}<K_{f \circ g}<K_{g}$

Corollary 7.2 Under assumption (7.1), the system $\{f, g\}$ has a 2 -periodic cycle for $f, g \in \mathcal{W}$.

Theorem 7.3 [20](Theorem 2.1, p. 47) [11](Corollary 2.4) Let $x^{*}$ be a fixed point of a continuous map on the compact interval $I=[a, b]$. Then $x^{*}$ is globally asymptotically stable relative on $(a, b)$ if and only if $f^{2}(x)>x$ for $x<x^{*}$ and $f^{2}(x)<x$ for $x>x^{*}$, for all $x \in(a, b)$.

Theorem 7.4 Let $f, g \in \mathcal{F}$ with $K_{f}<K_{g}$ and let $h=g \circ f$. We also assume that $g(x)>f(x)$ on $\left[K_{f}, \infty\right)$. Then the following statements hold true:

(i) if $C_{f}>K_{g}$, where $C_{f}$ is the critical point of $f$, then $K_{g \circ f}$ is asymptotically stable under $h$,

(ii) if $C_{f}<K_{g}$ and $h^{2}\left(C_{f}\right)>C_{f}$, then $K_{g \circ f}$ is asymptotically stable under $h$.

Proof

(i) Since $h^{\prime}(x)=g^{\prime}(f(x)) f^{\prime}(x)$, it follows that the critical point of $h$ are $C_{f}$ and all the points in the set $\left\{f^{-1}\left(C_{g}\right)\right\}$. Since $g(x)>f(x)$ on $\left[K_{f}, \infty\right),\left\{f^{-1}\left(C_{g}\right)\right\}=\emptyset$. Thus $C_{f}=C_{h}>K_{g}$. By Lemma 7.1, $C_{h}>K_{g \circ f}$. Hence $h \in \mathcal{F}$ and the conclusion follows.

(ii) Now assume that $C_{f}<K_{g}$ and $h^{2}\left(C_{f}\right)>C_{f}$. If $C_{f} \geq K_{g \circ f}$ we are done as in 1 . So let us assume that $C_{h}=C_{f}<K_{g \circ f}$. Hence $h\left(C_{h}\right)>K_{g \circ f}$ and $h^{2}\left(C_{h}\right)<K_{g \circ f}$. From the assumption $C_{h}<h^{2}\left(C_{h}\right)<K_{g \circ f}$. Thus in the invariant set $\left[C_{h}, h\left(C_{h}\right)\right]$, we have $h^{2}(x)>x$ if $x<K_{g \circ f}$ and $h^{2}(x)<x$ if $x>K_{g \circ f}$. Hence by Theorem 7.3 , we conclude that the fixed point $K_{g \circ f}$ is asymptotically stable.

Lemma 7.5 Suppose that assumption (6.6) holds. Then for any $K, f(x)$ in (6.4) belongs to $\mathcal{F}$.

Proof The proof follows from the discussion in Section 6. 
THEOREM 7.6 Let $\{f, g\}=\mathcal{W}$ be a 2 -periodic system, where $f, g$ as defined in (6.4) with carrying capacities $K_{f}, K_{g}$, respectively. Then under the assumption of theorem 7.4 , System $\mathcal{W}$ has an asymptotically stable 2 -periodic cycle, $\left\{K_{g \circ f}, f\left(K_{g \circ f}\right)\right\}$.

Proof The proof follows from 7.4 .

Acknowledgment: We would like to thank Rafael Luis for creating the graphs in this paper.

\section{Reference}

[1] W.C. Allee, The Social Life of Animals, William Heinemann, London, 1938.

[2] W.C. Allee, A.E. Emerson, O. Park, T. Park \& K.P. Schmidt, Principles of animal ecology, Saunders, Philadelphia, 1949.

[3] P. Bezandry, T. Diagana and S. Elaydi, On the stochastic Beverton-Holt equation with survival rates, J. Diff. Eq. \& Appl., 14 (2008), 175-190.

[4] F. Courchamp, L. Berec and J. Gascoigne, Allee Effects, Oxford University Press, Oxford, 2008.

[5] J.M. Cushing, The Allee effect in age-structured population dynamicsin T. Hallam, L. Gross, and S. Levin (Eds.), Mathematical Ecology, Springer-Verlag (1988), 479-505.

[6] J.M. Cushing, and S.M. Henson, Global dynamics of some periodically forced, monotone difference equations, J. Difference Equ.\& Appl., 7 (2001), 859-872.

[7] J.M. Cushing, and S.M. Henson, A periodically forced Beverton-Holt equation, J. Difference Equ. \& Appl., 8 (2002), 1119-1120.

[8] Elaydi, S., Discrete Chaos: With Applications in Science and Engineering, Second Edition, Chapman \& Hall/CRC, Boca Raton, 2008.

[9] S. Elaydi, and R. Sacker, Global stability of periodic orbits of nonautonomous difference equations and population biology, J. Differential Equations 208 (2005), 258-273.

[10] S. Elaydi, and R. Sacker, Global stability of periodic orbits of nonautonomous difference equations in population biology and the Cushing-Henson conjectures, Proceedings of the eighth International Conference on Difference Equations and Applications, Brno, 2003. 113-126, Chapman \& Hall/CRC, Boca Raton, FL, 2005.

[11] S. Elaydi, and R. Sacker, Basin of attraction of periodic orbits of maps in the real line, J. Difference Equ. \& Appl., Vol. 10 (2004), 881-888.

[12] S. Elaydi, and R. Sacker, Nonautonomous Beverton-Holt equations and the Cushing-Henson conjectures, J. Difference Equ. \& Appl., 11 (2005), 337-347.

[13] C. Haskell, R.J. Sacker, The Stochastic Beverton-Holt Equation and the M. Neubert Conjecture, J. Dynamics and Differential Equations, 17 (No. 4) (2005), pp. 825-842.

[14] S.M. Henson, Multiple attractors and resonance in periodically forced population models, Physica D, 40(2000), pp. 33-49.

[15] S.R.-J. Jang, Allee effects in a discrete-time host-parasitoid model. J. Difference Equ. \& Appl., 12 (2006), $165-181$.

[16] V.L. Kocic, A note on the nonautonomous Beverton-Holt model, J. Difference Eq. \& Appl., 11, Numbers 4-5,(2005), 337-347.

[17] R. Kon, A note on attenuant cycles of population models with periodic carrying capacity, J. Difference Equ. \& Appl., 10(8) (2004), 791-793.

[18] Jia Li, Baojun Song, and Xiaohong Wang, An extended discrete Ricker population model with allee effects. J. Difference Equ. \& Appl., 13 (2007), 309-321.

[19] Robert J. Sacker, A note on periodic Ricker maps, J. Difference Eq. \& Appl., 13(1):89-92, January 2007.

[20] A. N. Sharkovsky, Yu. L. Maistrenko and E. Yu. Romanenko, Difference equations and their applications, Kluwer Academic Publishers, London, 1993.

[21] S. J. Schreiber, Allee effects, extinctions, and chaotic transients in simple population models, Theoretical Population Biology 64 (2003), 201-209.

[22] P.A. Stephens, W.J. Sutherland \& R.P. Freckleton, 'What is the Allee Effect?', Oikos 87 (1999), 185-90.

[23] P.A. Stephens, \&W.J. Sutherland, Vertebrate mating systems, Allee effects and conservarion. In M. Apollonio, M. Festa-Bianchet and D. Mainardi, eds. Vertebrate mating systems, pp. 186-213, World Scientific Publishing, Singapore, 2000.

[24] Abdul-Aziz Yakubu, Allee effects in discrete-time SIUS epidemic models with infected newborns. J. Difference Equ. \& Appl., 13 (2007), 341-356.

[25] S. Zhou, Y. Liu, and G.Wang, The stability of predator-prey systems subject to the Allee effects. Theoretical Population Biology, Vol. 67 (2005), 23-31. 